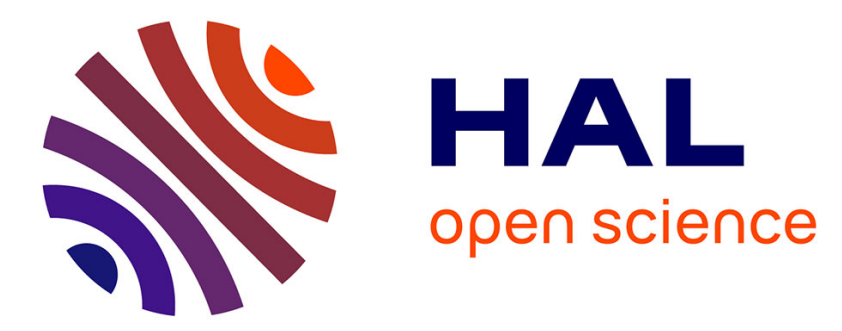

\title{
Spectral pulse synthesis in large-scale ultrafast coherent combining systems
}

F Guichard, Marc Hanna, L Lombard, Y Zaouter, C Hönninger, Fanny Morin, Frédéric Druon, E Mottay, Patrick Georges

\section{- To cite this version:}

F Guichard, Marc Hanna, L Lombard, Y Zaouter, C Hönninger, et al.. Spectral pulse synthesis in large-scale ultrafast coherent combining systems. The European Physical Journal. Special Topics, 2015, 224, pp.2545. 10.1140/epjst/e2015-02563-y · hal-01306661

\section{HAL Id: hal-01306661 \\ https://hal-iogs.archives-ouvertes.fr/hal-01306661}

Submitted on 25 Apr 2016

HAL is a multi-disciplinary open access archive for the deposit and dissemination of scientific research documents, whether they are published or not. The documents may come from teaching and research institutions in France or abroad, or from public or private research centers.
L'archive ouverte pluridisciplinaire HAL, est destinée au dépôt et à la diffusion de documents scientifiques de niveau recherche, publiés ou non, émanant des établissements d'enseignement et de recherche français ou étrangers, des laboratoires publics ou privés. 


\title{
Spectral pulse synthesis in large-scale ultrafast coherent combining systems
}

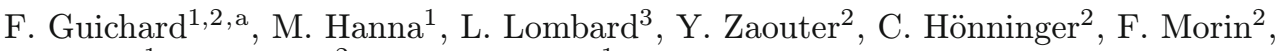 \\ F. Druon ${ }^{1}$, E. Mottay ${ }^{2}$, and P. Georges ${ }^{1}$ \\ 1 Laboratoire Charles Fabry, Institut d'Optique, CNRS, Université Paris-Sud, \\ 2 Av. Augustin Fresnel, 91127 Palaiseau Cedex, France \\ 2 Amplitude Systèmes, 11 avenue de Canteranne, Cité de la Photonique, 33600 Pessac, \\ France \\ 3 Office National d'Etudes et de Recherches Aérospatiales, Département d'Optique \\ Théoriques et Appliquée, Chemin de la Hunière, 91761 Palaiseau, France
}

\begin{abstract}
We propose the use of coherent spectral synthesis to reach shorter pulse durations in the context of massively parallel femtosecond coherent combining laser systems. A preliminary experiment that performs spectral synthesis with two femtosecond fiber chirped-pulse amplifiers is presented, and shows that a significant decrease of the amplified pulse duration compared to single-band amplification is achieved. We also discuss possible implementation of spectral synthesis in a large-scale coherent combining setup.
\end{abstract}

\section{Introduction}

The quest of a laser system able to generate both high average and high peak powers is one of the major trends of current research in the laser technology area. A wide range of scientific and societal applications would benefit from such systems, including high power extreme ultra-violet sources for photolithography, compact particle accelerators, and Higgs boson factories. The Coherent Amplifying Network (CAN) laser architecture has been designed to achieve both high average and high peak power. Proposed by the ICAN consortium [1] and based on early demonstrations of coherent combining in the femtosecond regime [2,3], this new laser concept involves coherently combining a large number of fiber laser channels. This concept takes advantage of the numerous desirable properties of fiber amplifiers: good thermal handling, high optical-to-optical efficiency, and excellent output beam quality. However, in high energy femtosecond fiber amplifiers, the achievable pulsewidth is limited to around $300 \mathrm{fs}$, mostly due to the gain profile of Yb-doped fibers which presents a strong peak at $1030 \mathrm{~nm}$ with a $\sim 40 \mathrm{~nm}$ bandwidth. This spectroscopic property induces an important limitation on the achievable bandwidth during high-gain amplification, a phenomenon denoted as gain narrowing. The typical available output bandwidth is

a e-mail: florent.guichard@institutoptique.fr 
around $12 \mathrm{~nm}$ in 10 to $20 \mathrm{~dB}$ low-gain amplifiers [4]. This pulsewidth limitation can hinder the use of an CAN system for some applications. As an example, preliminary laser specifications proposed by experts in the field of particle acceleration mentions pulsewidths of $60 \mathrm{fs}$ [5], which is clearly out of the range of current Yb-doped fiber amplifiers. In most cases, the generation of shorter pulses relaxes the specification on other critical parameters such as pulse energy and average power. In this context it is therefore paramount to devise laser architectures able to generate the shortest pulsewidth, while retaining the original framework of a massively parallel fiber amplifier network.

The possibility of combining ultrafast pulses with different spectral content to synthesize much shorter pulses was suggested in 1990 [6], and demonstrated in the context of few-cycle pulses recently [7]. This idea requires that the combined spectrum exhibits optical coherence across the entire spectral content, and therefore that the relative phase between the initial pulses can be controlled. As such, pulse synthesis requires both coherent combining (phase control between the pulses to be combined) and spectral combining (an optical element that allows the spatial combination of different central wavelengths). In this contribution we propose to use pulse synthesis in the context of a massively parallel coherent amplification network. A proof-of-concept experiment is presented and shows a significant improvement in pulsewidth for a femtosecond fiber amplifier system implementing pulse synthesis with two channels [8]. As an additional coherent combining step, the integration of pulse synthesis in an CAN system is natural, and should even provide additional benefits such as pulse shaping capabilities. We also discuss how pulse synthesis could be implemented and other advantages of this promising laser source architecture.

\section{Proof-of-principle experiment}

Here, we report on the development of a two-channel pulse synthesizer able to amplify two distinct parts of an incoming spectrum in order to mitigate gain narrowing. For gain values larger than $30 \mathrm{~dB}, 130 \mathrm{fs}$ pulses with a spectral width of $19 \mathrm{~nm}$ are generated in microstructured large mode area (LMA) Yb-doped fibers, at an average power level of $10 \mathrm{~W}$ and a repetition rate of $35 \mathrm{MHz}$. These results are compared to a single amplifier setup to quantify the benefits of spectral coherent combing to mitigate gain narrowing.

The experimental setup is presented in Fig. 1 (left) and starts with an air-cooled Yb:KYW oscillator generating Fourier-transformed-limited 270 fs pulses centered at $1030 \mathrm{~nm}$ at $35 \mathrm{MHz}$ repetition rate. Output pulses are sent into a 1-m length standard polarization-maintaining passive fiber to generate a $50 \mathrm{~nm}$ spectrum through self-phase modulation. It is followed by a 400 ps grating-based stretcher designed to support $50 \mathrm{~nm}$ bandwidth pulses. The coherent spectral combining setup is a MachZehnder interferometer, where input/output couplers are replaced by two identical sharp-edge dichroic mirrors to ensure proper spectral separation and recombination. A fiber-pigtailed $\mathrm{LiNbO}_{3}$ integrated phase modulator is inserted in the reflected interferometer arm (short-wavelength arm, SWA), and followed by a $2 \mathrm{~m}$-long Yb-doped double-clad polarization-maintaining 40/200 $\mu \mathrm{m}$ LMA fiber amplifier. The phase modulator is driven by a feedback loop to ensure active stabilization of the relative optical phase between the two interferometer arms. In the second arm (transmitted arm or long-wavelength arm, LWA), $2.4 \mathrm{~m}$ length of passive single-mode fiber, a free-space delay line and another $2 \mathrm{~m}$ Yb-doped double-clad 40/200 $\mu \mathrm{m}$ LMA fiber amplifier are implemented. The aim of the single-mode fiber is to compensate the second-order phase accumulated in the short-wavelength arm through propagation in the phase modulator and thus match the group-velocity dispersion of both arms 

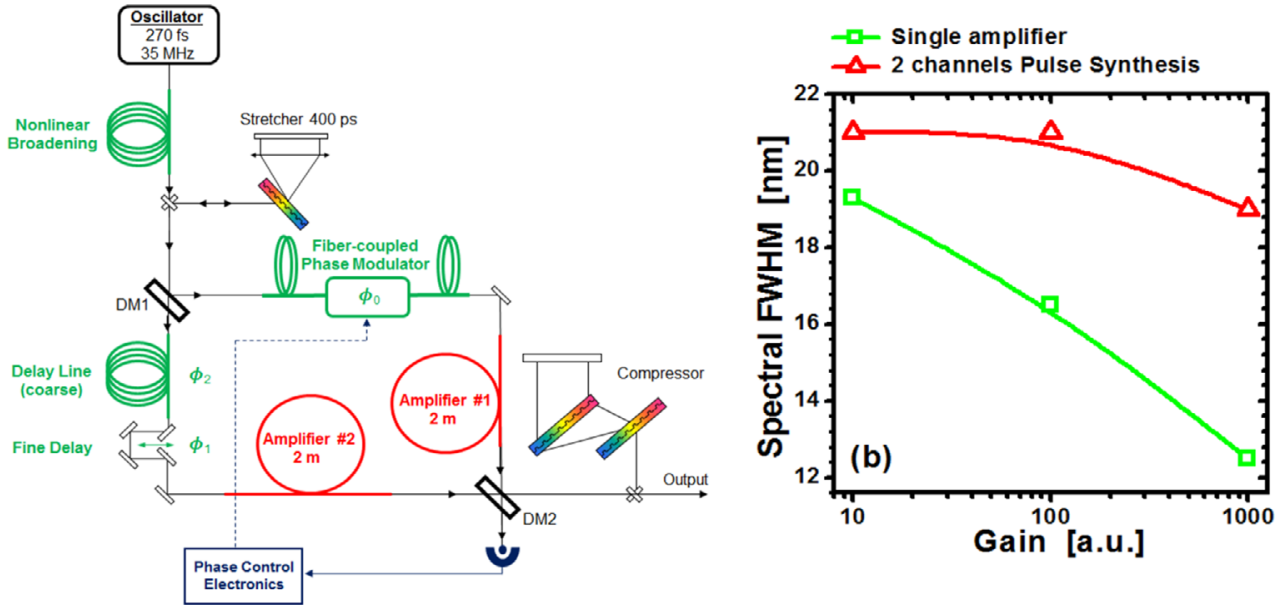

Fig. 1. Left: experimental setup of the pulse synthesis experiment. DM: Dichroïc mirror. Фn: nth order phase term correction. Right: Spectrum FWHM evolution for different gain levels in the two-channel (red-curve) and one-channel (green curve) configurations.

while the delay line acts as a fine delay matching element. Finally, the output of each amplifier is collimated and the optical paths from the LMA fibers end to the combining dichroic mirror are set as close as possible in order to match the spatial profiles and wavefronts of both beams. The beam is then sent to a grating-based compressor.

The spectral characteristics of the combined output are first studied as a function of gain value, and compared to a single amplifier setup to quantify the benefit of spectral synthesis with regard to gain narrowing. The spectral FWHM of both configurations as a function of gain are plotted in Fig. 1 (right). The spectral FWHM is clearly larger for the two-channel systems, highlighting the main advantage of spectral coherent combining. Gain narrowing is mitigated because narrower spectra are seeded to each amplifier, and pump levels can be adjusted to generate the largest bandwidth possible.

After careful adjustment of the pump power levels to match the average power of both arms, the obtained combined average power is $10 \mathrm{~W}$ at $35 \mathrm{MHz}$ repetition rate. At this power level, corresponding to a gain of $30 \mathrm{~dB}$, the combined spectrum exhibits a FWHM of $19 \mathrm{~nm}$, which outperforms any reported high-gain Yb-doped linear CPA fiber amplifier around $1030 \mathrm{~nm}$. Corresponding spectra are shown in Fig. 2 (left), as well as the stretched spectrum and the amplified spectra of each channel prior to recombination. The recombined spectrum fits with the superposition of the reflected and transmitted amplified spectra, that each exhibitsa FWHM of approximately $7 \mathrm{~nm}$, corresponding to a stretched pulse duration of approximately $60 \mathrm{ps}$ inside each fiber amplifier. These results can be compared to the spectral width when the full incident spectrum is seeded in a single amplifier at the same gain level. In such conditions despite having a much broader input spectrum to seed the amplifier with, the output spectrum FWHM is only $12 \mathrm{~nm}$.

Temporal characteristics are shown in Fig. 2 (right). The generated pulsewidths corresponding to each amplifying arm are roughly equal and minimized at the same compression point, indicating that the group velocity dispersion and spatial angular superposition mismatch between both beams are negligible. Indeed, minimum compressed pulse duration of both arms occurs for different compressor lengths when both 

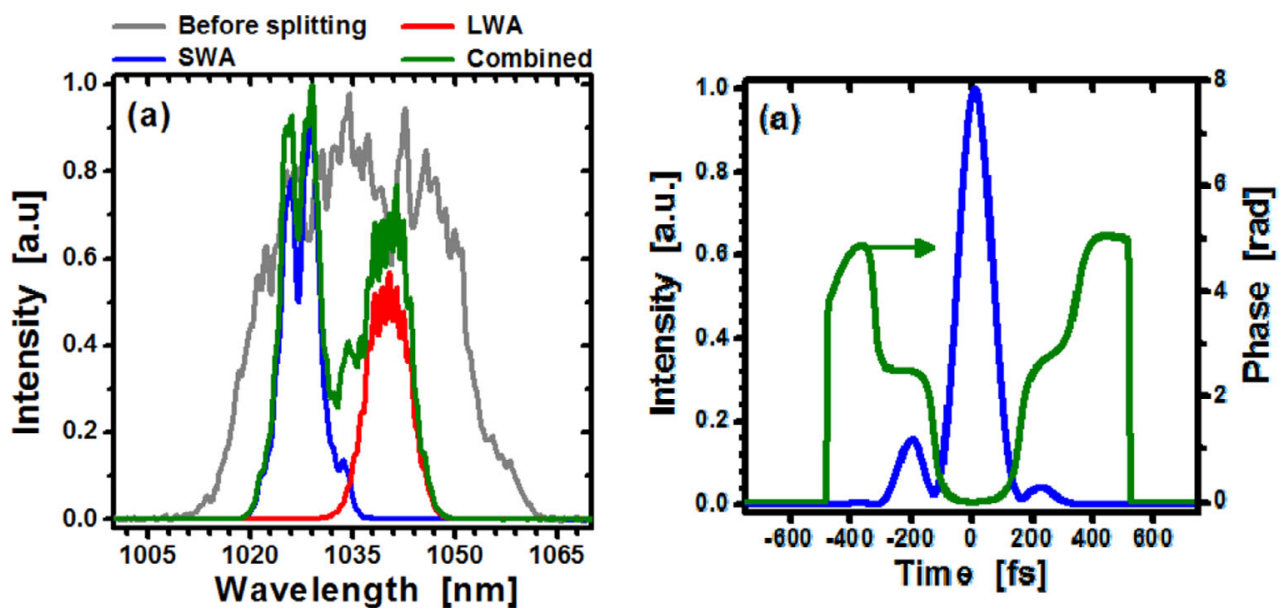

Fig. 2. Left: experimental stretched, combined, and separately amplified arm spectra at $10 \mathrm{~W}$ combined average power together with the output spectrum in single amplifier configuration. Right: FROG-retrieved temporal profile at $10 \mathrm{~W}$ combined average power.

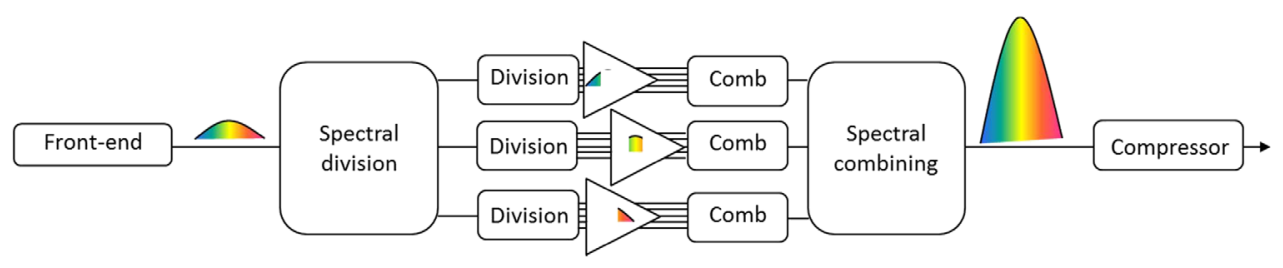

Fig. 3. Schematic of a possible pulse synthesis implementation in an CAN laser system.

beams are not perfectly superposed since different incident angles in the compressor unit change the induced spectral phase. An imperfect group-velocity dispersion matching between both arms leads to the same observation. A second-harmonic generation frequency-resolved optical gating (FROG) measurement reveals a combined pulse duration of $130 \mathrm{fs}$. Overall compression quality is satisfactory as indicated by the Fourier transform-limited pulse duration of the recombined spectrum, equal to $115 \mathrm{fs}$. The time-bandwidth product of the synthesized pulse is 0.70 , due to the two-lobe spectrum, and the temporal Strehl ratio is 0.79 .

\section{Pulse synthesis in a CAN laser}

The proof-of-principle experiment clearly demonstrates the advantage of the spectral pulse synthesis architecture in terms of achievable pulsewidth. It is clear that in order to fully take advantage of the pulse synthesis geometry, the amplifiers in each branch must exhibit optical gain bandwidths that are shifted from one another. This can be achieved, for example, by controlling the population inversion level of preamplifier stages inserted in each arm by varying the length of active fiber and the pumping power. It should be noted that this technique does not allow pulse energy scaling if the stretcher / compressor size is kept constant, since the pulse durations inside each spectral arm are lower than the total stretched pulse duration. Here, the quantity that is really scaled is the final peak power, since at constant energy, achieved 
pulse durations are shorter. We believe that a moderate number of spectral channels, ranging from 4 to 8, should allowa full coverage of the gain bandwidth in Yb-doped fibers, resulting in the generation of pulsewidths well below $100 \mathrm{fs}$. A possible way to implement the spectral division / recombination in the context of an CAN system is depicted in Fig. 3. Given the relatively small number of spectral channels needed, the recombination could be performed in a filled aperture configuration, outside the energy-scaling coherent combining steps, although system aspects details are still at an early stage of development.

The authors acknowledge the financial support of Agence Nationale de la Recherche through the MultiFemto project and European Union through the ICAN project. This work is supported by a public grant from the "Laboratoire d'Excellence Physics Atom Light Mater"

(LabEx PALM) overseen by the French National Research Agency (ANR) as part of the "Investissements d'Avenir" program (reference: ANR-10-LABX-0039).

\section{References}

1. G. Mourou, B. Brocklesby, T. Tajima, J. Limpert, Nat. Photon. 7, 258 (2013)

2. E. Seise, A. Klenke, J. Limpert, A. Tünnermann, Opt. Exp. 18, 27827 (2010)

3. L. Daniault, M. Hanna, L. Lombard, Y. Zaouter, E. Mottay, D. Goular, P. Bourdon, F. Druon, P. Georges, Opt. Lett. 36, 621 (2011)

4. L. Kuznetova, F.W. Wise, S. Kane, J. Squier. Appl. Phys. B 88, 515 (2007)

5. W. Leemans, W. Chou, M. Uesaka, ICFA Beam Dynamics Newsletter 56 (2011)

6. T.W. Hänsch, Opt. Comm. 80, 71 (1990)

7. S.-W. Huang, G. Cirmi, J. Moses, K.-H. Hong, S. Bhardwaj, J.R. Birge, L.-J. Chen, E. Li, B. Eggleton, G. Cerullo, F.X. Kärtner, Nat. Photon. 5, 475 (2011)

8. F. Guichard, M. Hanna, L. Lombard, Y. Zaouter, C. Hönninger, F. Morin, F. Druon, E. Mottay, P. Georges, Opt. Lett. 38, 5430 (2013) 